\title{
EQUAÇÕES DE INTENSIDADE DE CHUVAS PARA O ESTADO DO MARANHÃO
}

\author{
Alcinei Ribeiro Campos ${ }^{1}$, Glenio Guimarães Santos², João Carlos Rocha dos Anjos ${ }^{3}$, Diane Cristina Stefanoski
}

Zamboni $^{4}$, Jaurene Moura Fé Moraes ${ }^{5}$

\begin{abstract}
RESUMO
Estudos da distribuição espacial e temporal das chuvas, com determinação das curvas de intensidade-duraçãofrequência, são de extrema importância para segurança no planejamento e dimensionamento de projetos hidráulicos e conservação do meio ambiente. . Atualmente, o estado do Maranhão disponibiliza de poucas informações relacionadas às curvas de intensidade-duração-frequência das chuvas, necessárias para caracterização dessa variável hidrológica para seus diversos municípios. Diante desse contexto, objetivou-se ajustar os parâmetros das curvas de intensidadeduração-frequência para 163 estações pluviométricas localizadas no estado do Maranhão. Os dados foram obtidos do banco de dados da Agencia Nacional de Águas. Para ajuste das curvas de intensidade-duração-frequênciautilizou-se o método de desagregação das chuvas, usando o modelo probabilístico de Gumbel para estimar os valores máximos em diferentes tempos de durações e período de retorno. De posse desses valores, os parâmetros $\boldsymbol{K}, \boldsymbol{a}, \boldsymbol{b}$ e $\boldsymbol{c}$ das curvas intensidade-duração-frequência foram ajustados pelo método dos mínimos quadrados. Os parâmetros $\boldsymbol{K}, \boldsymbol{a}, \boldsymbol{b}$ e $\boldsymbol{c}$ das curvas intensidade-duração-frequência apresentaram bons ajustes, com 99,7\% das equações apresentando $r^{2}$ superior a 0,98 .
\end{abstract}

Palavras-chave: Chuvas extremas; modelos probabilísticos; período de retorno

\section{ABSTRACT}

\section{RAINFALL INTENSITY EQUATIONS FOR THE STATE OF MARANHÃO, BRAZIL}

Studies of spatial and temporal distribution of rainfall are of extreme importance for security in planning and execution of hydraulic projects and environmental conservation, so work to determine the intensity-duration-frequency curves of rainfall are indispensable for the planning and construction of hydraulic works. Currently the state of Maranhão provides little information related to the curves of intensity-duration-frequency of rainfall, so studies are necessary to develop the curves for its various municipalities. In this context the objective of this work was to determine the parameters of the intensity-duration-frequency equation for 163 rainfall stations located in the state of Maranhão. Data were obtained from the National Water Agency database. For adjustment of intensity-duration-frequency curves the rainfall breakdown method was used, considering the probabilistic model of Gumbel for estimating the maximum values in different time durations and return period. Upon acquisition of these values the parameters $\mathrm{K}$, a, b and c of the intensity-duration-frequency curves were adjusted by the least squares method. The parameters $\mathrm{K}$, a, b and c of the intensity-duration-frequency curves show good adjust, with $99.7 \%$ of curves presenting $\mathrm{r}^{2}$ greater than 0.98 .

Keywords: Extreme rainfall, Probabilistic models end Return period

\section{Recebido para publicação em 15/04/2015. Aprovado em 19/08/2015.}

1 - Engenheiro Agrônomo. Doutorando no Programa de Pós-graduação Ciência do Solo. PPGCS /UFRGS. alcineicampos@gmail.com

2 - Engenheiro Agrônomo. Doutor em solo e água. Professor da Universidade Federal de Goiás - UFG. gleniogm@gmail.com

3 - Engenheiro Agrônomo. Mestre em agronomia. CPCE/UFPI/Bom Jesus - PI. agrojoaocarlos@gmail.com

4 - Engenheiro Agrônomo. Mestre em Solos e Nutrição de Plantas. CODEMA/Água Boa-MT.diane stefanoski@hotmail.com

5 - Bióloga, Mestre em agronomia, CPCE/UFPI/Bom Jesus - PI. jaurene.moura@hotmail.com 


\section{INTRODUÇÃO}

No Brasil, nos últimos anos, tem havido grande expansão na ocupação de novas áreas seja para construção civil, a exemplo da construção de infraestrutura ou expansão urbana, mas também para exploração com agropecuária a exemplo das novas áreas que vem sendo utilizadas no cerrado. Essas ocupações nem sempre tem levado em consideração todas as informações necessárias a seu planejamento, a exemplo são as informações sobre a capacidade de uso das terras para tais fins, assim como conhecimento das informações sobre a distribuição espacial e temporal das chuvas, principalmente no que se refere às chuvas intensas.

Segundo Cecílio et al. (2009) e Santos et al. (2010), as chuvas intensas são aquelas capazes de causar inundações e, consequentemente, diversos prejuízos, tanto em áreas urbanas quanto em áreas agrícolas. Dessa forma, a sua quantificação temporal e espacial é de crucial importância em estudos relacionados a dimensionamento de projetos hidráulicos, como obras para o controle em áreas agrícolas e urbanas e o armazenamento e fornecimento de água, tanto para irrigação, indústria, abastecimento doméstico, quanto para dessedentação animal (RODRIGUES et al. 2008. MORUZZI et al. 2009).

De acordo com Damé et al. (2008), a quantificação das chuvas intensas pode ser realizada por meio do emprego de modelos matemáticos empíricos, denominados de curvas de intensidadeduração-frequência, também conhecidas como curvas ou equações IDF. O ajuste dessas equaçõesé realizado de forma empírica, a partir do ajuste de parâmetros extraídos de dados pluviométricos para cada estação e local (OLIVEIRA et al., 2005).

No Brasil, os primeiros estudos para ajuste de equações IDF foram desenvolvidos por Pfafstetter (1957) e Denardin e Freitas (1982), em que foram ajustadas as equações IDF para 80 estações distribuídas por todo o país. Dentre os trabalhos mais recentes, destacam-se os realizados por Cecílio et al. (2009), Santos et al. (2010), Aragão et al. (2013) e Campos et al. (2014) para os estados do Espirito Santo, Sergipe, Mato Grosso do Sul e
Piauí respectivamente.

Para o estado do Maranhão, verifica-se que há uma carência de trabalhos relacionados às curvas de IDF, tendo em vista sua grande área territorial e a diversidade climática existente entre a parte mais ao litoral e a porção localizada dentro do bioma Amazônico. A distribuição de precipitação aumenta da direção sudoeste para noroeste do Estado (PINTO et al., 2011). Na parte mais ao sudoeste do Estado encontra se o bioma Cerrado que apresenta uma precipitação em torno dos 1100 $\mathrm{mm}$ anuais, na região mais a nordeste, já no bioma Amazônia, ocorre precipitação que pode superar os $2000 \mathrm{~mm}$ anuais, assim pode se observar que ocorre grande variação no volume de chuvas dentro do Estado (PINTO et al., 2011). No entanto, apesar da existência de grande variação na distribuição das chuvas no Estado, pouco se conhece sobre sua intensidade, duração e frequência, sendo que os únicos trabalhos existentes foram realizados por Dernadin e Freitas (1982), quando foram ajustadas as curvas de IDF para três municípios do Estado (São Luís, Barra do Corda e Turiaçu).

Nesse sentido, tendo em vista a variação nos fenômenos meteorológicos no Estado e a importância do conhecimento das equações que relacionam a intensidade, duração e frequência das precipitações e a inexistência desses modelos matemáticos para a maioria dos municípios do estado do Maranhão, este trabalho teve como objetivo ajustar os parâmetros da equação de intensidade-duração-frequência para 163 estações pluviométricas localizadas no estado do Maranhão.

\section{MATERIAL E MÉTODOS}

Para a realização deste trabalho foram utilizados dados pluviométricos coletados por 163 estações pluviométricas localizadas no estado do Maranhão (Figura 1), disponíveis no banco de dados da Agência Nacional de Águas (ANA), em seu sítio eletrônico.

Para cada estação foram ajustados os parâmetros $\boldsymbol{K}, \boldsymbol{a}, \boldsymbol{b}$, e $\boldsymbol{c}$ da equação de intensidade, duração e frequência (Equação 1), por meio de regressão linear. 

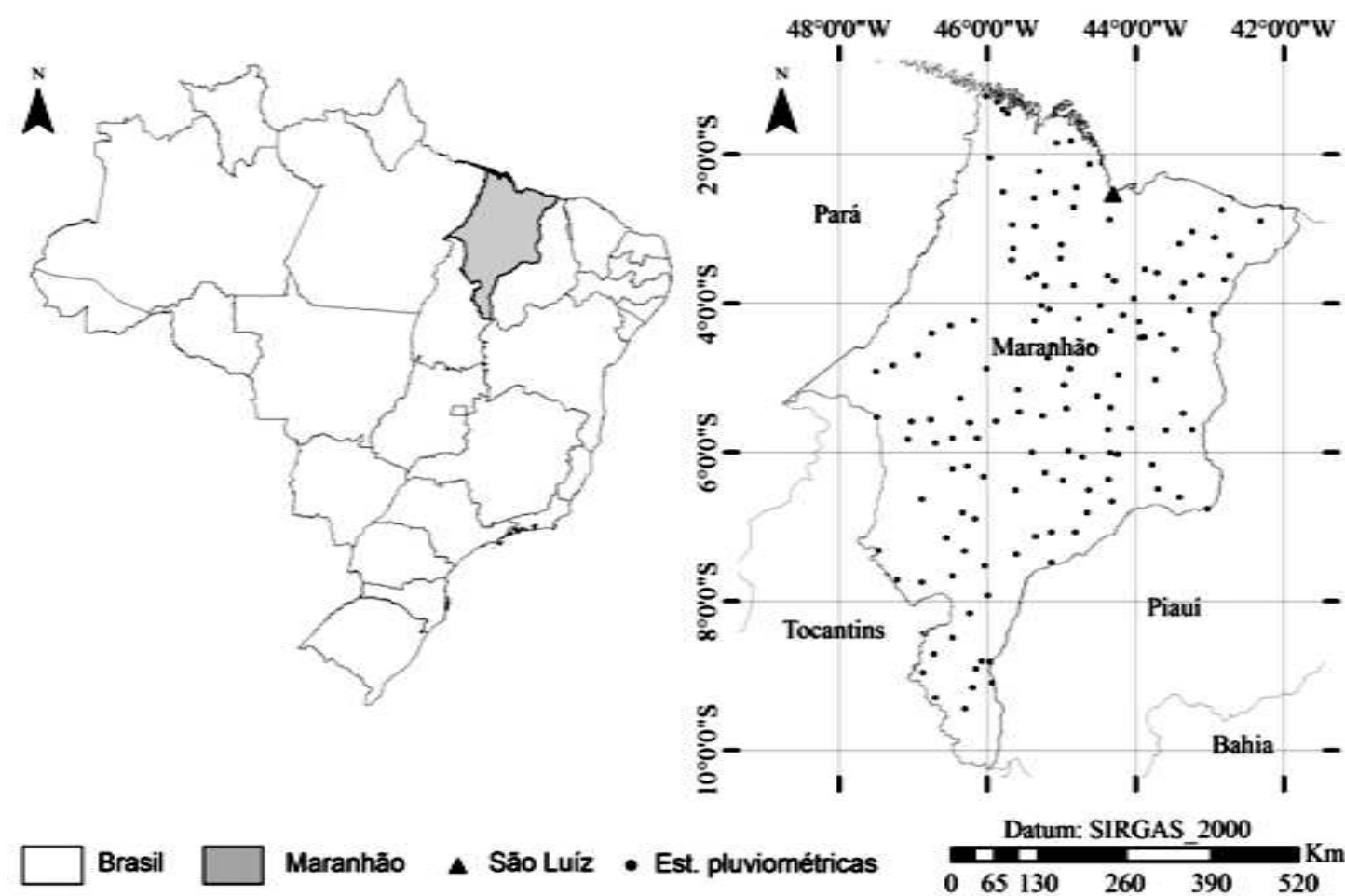

Figura 1. Distribuição espacial das 163 estações pluviométricas no estado do Maranhão.

$$
\operatorname{Im}=\frac{\left(K^{*} \mathrm{TR}\right)^{a}}{(\mathrm{t}+b)^{\mathrm{c}}}
$$

em que,

Im - intensidade máxima média de precipitação, $\mathrm{mm} \mathrm{h}^{-1}$;

$\boldsymbol{T R}$ - período de retorno, anos;

$\boldsymbol{t}$ - duração da precipitação, min.; e

$\boldsymbol{K}, \boldsymbol{a}, \boldsymbol{b}$ e $\boldsymbol{c}$ - parâmetros ajustados com base nos dados pluviométricos da localidade.

Inicialmente, foi realizada uma análise de consistência das séries de dados de cada estação, sendo que, para cada uma, foram selecionados os anos que continham menores números de falhas nos dados, e descartadas as estações que ficaram com menos de 15 anos na série de dados.Após análise de consistência das séries de dados, para cada estação, foram selecionadas as chuvas extremas de um dia, a partir das quais, por meio do emprego do método da desagregação das chuvas de um dia, foram geradas chuvas máximas para duração de 5 , $10,15,20,25,30,60,360,480,600,720$ e 1.440 min., empregando os coeficientes propostos por Cetesb (1979) (Quadro 1).

Depois de realizada a desagregação das chuvas em durações inferiores,foram estimados os eventos máximos para cada duração e períodos de retorno (TR) de 5, 10, 25, 50 e 100 anos, aplicando o modelo probabilístico de Gumbel (MELLO; SILVA 2005), conforme Equação (2).

$$
h_{T R}=a-b \operatorname{Ln}\left[\operatorname{Ln}\left(\frac{T R}{T R-1}\right)\right]
$$

em que,

$\boldsymbol{h}_{T R^{-}}$altura pluviométrica associada a um período de retorno $\boldsymbol{T R}, \mathrm{mm}$; e

$\boldsymbol{a}$ e $\boldsymbol{b}$ - parâmetros da distribuição de Gumbel, obtidos pelo método dos momentos (Equações 3 e 4). 
Quadro 1. Coeficientes de desagregação da precipitação para intervalos de tempos menores

\begin{tabular}{cc}
\hline Intervalo de transformação & Coeficientes \\
\hline $1 \mathrm{dia} / 24 \mathrm{~h}$ & 1,14 \\
$1 \mathrm{dia} / 12 \mathrm{~h}$ & 0,85 \\
$24 \mathrm{~h} / 10 \mathrm{~h}$ & 0,82 \\
$24 \mathrm{~h} / 8 \mathrm{~h}$ & 0,78 \\
$24 \mathrm{~h} / 6 \mathrm{~h}$ & 0,72 \\
$24 \mathrm{~h} / 1 \mathrm{~h}$ & 0,42 \\
$1 \mathrm{~h} / 30 \mathrm{~min}$. & 0,74 \\
$1 \mathrm{~h} / 25 \mathrm{~min}$. & 0,91 \\
$1 \mathrm{~h} / 20 \mathrm{~min}$. & 0,81 \\
$1 \mathrm{~h} / 15 \mathrm{~min}$. & 0,70 \\
$1 \mathrm{~h} / 10 \mathrm{~min}$. & 0,54 \\
$1 \mathrm{~h} / 5 \mathrm{~min}$. & 0,34 \\
\hline
\end{tabular}

Fonte: Cetesb (1979).

$$
\begin{aligned}
& E(h)=a \pm 0,577 b \\
& \operatorname{VAR}(h)=1,645 b^{2}
\end{aligned}
$$

em que,

$\boldsymbol{E}(\boldsymbol{h})$ - valor esperado estimado pela média dos valores máximos anuais; e

$\boldsymbol{V A R}(\mathbf{h})$ - variância dos valores máximos anuais, estimada a partir da série de dados.

De posse dos valores das máximas precipitações para cada duração e período de retorno, realizou-se o ajuste dos parâmetros $(\boldsymbol{K}, \boldsymbol{a}, \boldsymbol{b}$ e c $)$ da equação IDF (Equação 1) para cada estação, pelo método dos mínimos quadrados.

Para realizar o ajuste dos parâmetros empíricos da IDF, a Equação (1) foi dividida em duas novas equações (5 e 6):

$$
\begin{aligned}
& i=\frac{C}{(t+b)^{n}} \\
& C=K * T R^{a}
\end{aligned}
$$

em que,

$\mathrm{i}=$ intensidade estimada;

$\boldsymbol{t}=$ tempo em minutos,

$\boldsymbol{T R}=$ período de retorno em anos; e

$\boldsymbol{K}, \boldsymbol{a}, \boldsymbol{b}$ e $\boldsymbol{n}=$ parâmetros empíricos ajustados.

Em seguida, mediante a propriedade dos logaritmos, a Equação 5 foi transformada na equação da reta (Equação 7), da seguinte forma:

$$
\log i=\log C-n * \log (t+b)
$$

Após esse procedimento, foi calculado o logaritmo da intensidade máxima para cada duração $(\log i)$, em cada período de retorno. O valor de $\boldsymbol{b}$ foi obtido por tentativa, utilizandose análise de regressão aplicada aos valores dos logaritmos da intensidade $(\log i)$ para um dado período de retorno, com relação aos logaritmos das durações $(\log t)$, conforme proposto por Villela e Mattos (1975).

Dessa forma, com os valores de $\log \boldsymbol{i}$ e $\log (\boldsymbol{t}+\boldsymbol{b})$, foram gerados gráficos de dispersão, procedendo, na sequência, o ajuste dos coeficientes angular (n)

\section{REVENG


e linear $(\log C)$, para cada período de retorno. Os parâmetros $\boldsymbol{K}$ e $\boldsymbol{m}$ da equação IDF foram estimados pelo método dos mínimos quadrados, conforme a seguinte expressão (Equação 8):

$$
\log C=\log k+m * \log T R
$$

em que,

$\log \boldsymbol{K}$ e $\boldsymbol{m}$ - coeficientes linear e angular da reta, respectivamente; e

$\log T R$, período de retorno.

Para ajuste dos parâmetros $\boldsymbol{K}, \boldsymbol{a}, \boldsymbol{b}$ e $\boldsymbol{c}$, todas as equações citadas acima são implantadas em planilhas eletrônica Excell, nas quais são criadas rotinas por meio de macros para importar e realizar todo o processamento dos dados e o ajuste dos parâmetros da IDF. O uso de regressões para o ajuste pode acarretar na fixação do valor de alguns desses parâmetros, no entanto, geralmente resulta em equações com bons ajustes aos dados das quais foram derivadas (CAMPOS et al., 2014; SANTOS et al., 2009).

Após ajustados os parâmetros da IDF, foi realizada a espacialização dos parâmetros $\boldsymbol{K}$ e $\boldsymbol{a}$ para toda área territorial do Estado. A escolha desses parâmetros para espacialização se deu em função da sua variação entre as estações estudadas. A espacialização foi realizada com auxílio do software ArcGiz versão 10, no qual foi utilizando o método de interpolação Kriging, do tipo ordinária.

De posse dos parâmetros da IDF, foi realizada a estimativa das intensidades para duração de 10,760 e 720 minutos e período de retorno de 10 anos para todas as estações, seguida por sua espacialização, por meio do método de interpolação Kriging, do tipo ordinária (MELLO et al. , 2003).

\section{RESULTADO E DISCUSSÃO}

O ajuste dos parâmetros $(\boldsymbol{K}, \boldsymbol{a}, \boldsymbol{b}$ e $\boldsymbol{c})$ da IDF, determinado utilizando o modelo de regressão linear apresentou 99,7\% dos coeficientes de determinação superiores à 0,99 (Quadro 2), indicando confiabilidade dos dados climatológicos estudados.

Quadro 2. Parâmetros (K, $\boldsymbol{a}, \boldsymbol{b}$ e $\boldsymbol{c})$ da equação de intensidade-duração-frequência ajustados por regressão linear para 163 estações localizadas no estado do Maranhão.

\begin{tabular}{llccccccc}
\hline Código & Município & Latitude & Longitude & $\mathrm{K}$ & $\mathrm{a}$ & $\mathrm{b}$ & $\mathrm{c}$ & $\mathrm{r}^{2}$ \\
\hline 00146004 & Carutapera & $-1,217$ & $-46,017$ & 999,310 & 0,190 & 10 & 0,742 & 0,991 \\
00245007 & Turiaçu & $-2,505$ & $-45,784$ & 933,250 & 0,166 & 10 & 0,742 & 0,993 \\
00245008 & Viana & $-2,971$ & $-45,355$ & 813,950 & 0,136 & 10 & 0,742 & 0,995 \\
00245009 & Pinheiro & $-2,590$ & $-45,365$ & 876,600 & 0,162 & 10 & 0,742 & 0,993 \\
00245010 & Pinheiro & $-2,515$ & $-45,086$ & 971,180 & 0,146 & 10 & 0,742 & 0,994 \\
00145001 & Godofredo Viana & $-1,400$ & $-45,783$ & 1238,510 & 0,143 & 10 & 0,742 & 0,995 \\
00145002 & Luís Domingues & $-1,300$ & $-45,867$ & 909,290 & 0,177 & 10 & 0,742 & 0,992 \\
00145003 & Cururupu & $-1,850$ & $-45,067$ & 1691,220 & 0,199 & 10 & 0,742 & 0,991 \\
00145005 & Turiaçu & $-1,667$ & $-45,367$ & 876,400 & 0,179 & 10 & 0,742 & 0,992 \\
00145006 & Cândido Mendes & $-1,456$ & $-45,728$ & 1004,850 & 0,145 & 10 & 0,742 & 0,995 \\
00242000 & Barreirinhas & $-2,750$ & $-42,833$ & 967,390 & 0,126 & 10 & 0,742 & 0,996 \\
00242001 & Tutóia & $-2,767$ & $-42,283$ & 1159,310 & 0,183 & 10 & 0,742 & 0,992 \\
00242002 & Tutóia & $-2,902$ & $-42,313$ & 986,050 & 0,152 & 10 & 0,742 & 0,994 \\
00244000 & Bequimão & $-2,450$ & $-44,800$ & 692,310 & 0,211 & 10 & 0,742 & 0,990 \\
00244001 & Guimarães & $-2,133$ & $-44,617$ & 1391,870 & 0,187 & 10 & 0,742 & 0,992
\end{tabular}




\begin{tabular}{|c|c|c|c|c|c|c|c|c|}
\hline 00244003 & Rosário & $-2,950$ & $-44,250$ & 979,490 & 0,166 & 10 & 0,742 & 0,994 \\
\hline 00244010 & São Bento & $-2,717$ & $-44,833$ & 1235,380 & 0,147 & 10 & 0,742 & 0,994 \\
\hline 00244011 & Guimarães & $-2,129$ & $-44,607$ & 1115,840 & 0,129 & 10 & 0,742 & 0,995 \\
\hline 00244012 & São Bento & $-2,701$ & $-44,823$ & 1113,530 & 0,174 & 10 & 0,742 & 0,993 \\
\hline 00245001 & Monção & $-2,954$ & $-45,665$ & 998,160 & 0,136 & 10 & 0,742 & 0,995 \\
\hline 00245003 & Cândido Mendes & $-2,052$ & $-45,958$ & 886,750 & 0,153 & 10 & 0,742 & 0,994 \\
\hline 00342000 & Brejo & $-3,683$ & $-42,800$ & 683,600 & 0,210 & 10 & 0,742 & 0,990 \\
\hline 00342001 & Santa Quitéria do Maranhão & $-3,117$ & $-42,933$ & 642,540 & 0,205 & 10 & 0,742 & 0,990 \\
\hline 00643000 & São João dos Patos & $-6,500$ & $-43,700$ & 927,900 & 0,153 & 10 & 0,742 & 0,994 \\
\hline 00342009 & Santa Quitéria do Maranhão & $-3,365$ & $-42,724$ & 937,350 & 0,141 & 10 & 0,742 & 0,989 \\
\hline 00343001 & Vargem Grande & $-3,554$ & $-43,874$ & 927,260 & 0,143 & 10 & 0,742 & 0,995 \\
\hline 00343003 & Nina Rodrigues & $-3,459$ & $-43,899$ & 1470,280 & 0,313 & 10 & 0,742 & 0,979 \\
\hline 00343004 & Vargem Grande & $-3,595$ & $-43,709$ & 872,370 & 0,135 & 10 & 0,742 & 0,995 \\
\hline 00343005 & Urbano Santos & $-3,200$ & $-43,400$ & 930,470 & 0,163 & 10 & 0,742 & 0,993 \\
\hline 00343008 & Chapadinha & $-3,733$ & $-43,350$ & 1137,630 & 0,208 & 10 & 0,742 & 0,990 \\
\hline 00343009 & Mata Roma & $-3,626$ & $-43,112$ & 1074,730 & 0,140 & 10 & 0,742 & 0,995 \\
\hline 00343010 & Chapadinha & $-3,926$ & $-43,501$ & 969,170 & 0,131 & 10 & 0,742 & 0,995 \\
\hline 00343011 & Barreirinhas & $-3,044$ & $-43,237$ & 900,330 & 0,165 & 10 & 0,742 & 0,993 \\
\hline 00344003 & Cantanhede & $-3,633$ & $-44,400$ & 1003,230 & 0,128 & 10 & 0,742 & 0,996 \\
\hline 00344007 & Pirapemas & $-3,706$ & $-44,287$ & 925,760 & 0,130 & 10 & 0,742 & 0,995 \\
\hline 00344008 & Pirapemas & $-3,944$ & $-44,018$ & 1027,310 & 0,147 & 10 & 0,742 & 0,994 \\
\hline 00344011 & São Mateus do Maranhão & $-4,032$ & $-44,472$ & 989,240 & 0,156 & 10 & 0,742 & 0,994 \\
\hline 00344012 & Itapecuru Mirim & $-3,567$ & $-44,583$ & 1749,440 & 0,303 & 10 & 0,742 & 0,980 \\
\hline 00344013 & Vitória do Mearim & $-3,767$ & $-44,833$ & 992,890 & 0,167 & 10 & 0,742 & 0,993 \\
\hline 00345000 & Vitória do Mearim & $-3,771$ & $-45,218$ & 1034,190 & 0,153 & 10 & 0,742 & 0,994 \\
\hline 00345005 & Pindaré-Mirim & $-3,617$ & $-45,350$ & 969,840 & 0,112 & 10 & 0,742 & 0,996 \\
\hline 00345006 & Pindaré-Mirim & $-3,658$ & $-45,443$ & 874,180 & 0,157 & 10 & 0,742 & 0,991 \\
\hline 00345009 & Viana & $-3,217$ & $-45,000$ & 1131,880 & 0,134 & 10 & 0,742 & 0,995 \\
\hline 00345010 & Monção & $-3,233$ & $-45,617$ & 994,030 & 0,156 & 10 & 0,742 & 0,994 \\
\hline 00345011 & Monção & $-3,267$ & $-45,650$ & 1060,230 & 0,180 & 10 & 0,742 & 0,992 \\
\hline 00345012 & Cajari & $-3,403$ & $-45,011$ & 1091,940 & 0,151 & 10 & 0,742 & 0,994 \\
\hline 00345013 & Monção & $-3,424$ & $-45,669$ & 1053,170 & 0,148 & 10 & 0,742 & 0,994 \\
\hline 00346002 & Bom Jardim & $-4,231$ & $-46,179$ & 1030,620 & 0,151 & 10 & 0,742 & 0,994 \\
\hline 00442013 & Duque Bacelar & $-4,153$ & $-42,946$ & 959,840 & 0,134 & 10 & 0,742 & 0,995 \\
\hline 00443006 & Codó & $-4,459$ & $-43,877$ & 1015,080 & 0,137 & 10 & 0,742 & 0,995 \\
\hline 00443007 & Codó & $-4,464$ & $-43,931$ & 1009,020 & 0,164 & 10 & 0,742 & 0,993 \\
\hline 00443008 & Chapadinha & $-4,100$ & $-43,267$ & 925,980 & 0,126 & 10 & 0,742 & 0,996 \\
\hline 00443009 & Timbiras & $-4,250$ & $-43,950$ & 979,940 & 0,143 & 10 & 0,742 & 0,995 \\
\hline 00443012 & Aldeias Altas & $-4,626$ & $-43,468$ & 895,980 & 0,143 & 10 & 0,742 & 0,995 \\
\hline
\end{tabular}




\begin{tabular}{|c|c|c|c|c|c|c|c|c|}
\hline 00444000 & Bacabal & $-4,233$ & $-44,783$ & 1031,810 & 0,120 & 10 & 0,742 & 0,996 \\
\hline 00444001 & Coroatá & $-4,163$ & $-44,166$ & 946,670 & 0,149 & 10 & 0,742 & 0,994 \\
\hline 00444002 & Esperantinópolis & $-4,883$ & $-44,883$ & 1061,450 & 0,136 & 10 & 0,742 & 0,995 \\
\hline 00444003 & Gonçalves Dias & $-4,967$ & $-44,233$ & 904,900 & 0,137 & 10 & 0,742 & 0,995 \\
\hline 00444005 & Pedreiras & $-4,570$ & $-44,605$ & 924,490 & 0,147 & 10 & 0,742 & 0,994 \\
\hline 00444008 & Barra do Corda & $-5,101$ & $-44,961$ & 921,510 & 0,144 & 10 & 0,742 & 0,995 \\
\hline 00444012 & Bacabal & $-4,217$ & $-44,767$ & 1054,870 & 0,122 & 10 & 0,742 & 0,996 \\
\hline 00444013 & Coroatá & $-4,375$ & $-44,333$ & 927,260 & 0,145 & 10 & 0,742 & 0,994 \\
\hline 00445001 & Santa Luzia & $-4,031$ & $-45,271$ & 1092,450 & 0,154 & 10 & 0,742 & 0,994 \\
\hline 00445006 & Vitorino Freire & $-4,083$ & $-45,167$ & 1149,480 & 0,145 & 10 & 0,742 & 0,994 \\
\hline 00445007 & Lago da Pedra & $-4,743$ & $-45,181$ & 1052,200 & 0,162 & 10 & 0,742 & 0,993 \\
\hline 00445008 & Grajaú & $-4,886$ & $-46,011$ & 855,660 & 0,148 & 10 & 0,742 & 0,994 \\
\hline 00445009 & Lago da Pedra & $-4,561$ & $-45,126$ & 956,310 & 0,116 & 10 & 0,742 & 0,996 \\
\hline 00445010 & Vitorino Freire & $-4,241$ & $-45,358$ & 1108,660 & 0,144 & 10 & 0,742 & 0,995 \\
\hline 00446000 & Santa Luzia & $-4,304$ & $-46,494$ & 990,380 & 0,152 & 10 & 0,742 & 0,994 \\
\hline 00446001 & Santa Luzia & $-4,409$ & $-46,748$ & 823,760 & 0,173 & 10 & 0,742 & 0,993 \\
\hline 00446002 & Santa Luzia & $-4,698$ & $-46,939$ & 987,870 & 0,135 & 10 & 0,742 & 0,995 \\
\hline 00447002 & Imperatriz & $-4,841$ & $-47,274$ & 1130,320 & 0,150 & 10 & 0,742 & 0,994 \\
\hline 00447004 & Açailândia & $-4,921$ & $-47,497$ & 1162,790 & 0,146 & 10 & 0,742 & 0,994 \\
\hline 00543002 & Parnarama & $-5,484$ & $-43,359$ & 1031,570 & 0,229 & 10 & 0,742 & 0,988 \\
\hline 00543003 & Matões & $-5,700$ & $-43,233$ & 944,280 & 0,168 & 10 & 0,742 & 0,993 \\
\hline 00543004 & Buriti Bravo & $-5,709$ & $-43,587$ & 963,610 & 0,151 & 10 & 0,742 & 0,994 \\
\hline 00543006 & Caxias & $-5,033$ & $-43,733$ & 891,660 & 0,129 & 10 & 0,742 & 0,996 \\
\hline 00543011 & Passagem Franca & $-5,970$ & $-43,416$ & 1192,340 & 0,203 & 10 & 0,742 & 0,990 \\
\hline 00544000 & Fortuna & $-5,683$ & $-44,067$ & 771,610 & 0,159 & 10 & 0,742 & 0,994 \\
\hline 00544001 & Barra do Corda & $-5,983$ & $-44,900$ & 873,780 & 0,140 & 10 & 0,742 & 0,995 \\
\hline 00544004 & São Domingos do Maranhão & $-5,700$ & $-44,367$ & 827,180 & 0,150 & 10 & 0,742 & 0,994 \\
\hline 00544006 & Presidente Dutra & $-5,420$ & $-44,928$ & 865,770 & 0,157 & 10 & 0,742 & 0,994 \\
\hline 00544009 & Graça Aranha & $-5,405$ & $-44,336$ & 886,140 & 0,123 & 10 & 0,742 & 0,996 \\
\hline 00545001 & Itaipava do Grajaú & $-5,167$ & $-45,583$ & 768,420 & 0,185 & 10 & 0,742 & 0,992 \\
\hline 00545003 & Barra do Corda & $-5,500$ & $-45,217$ & 911,380 & 0,176 & 10 & 0,742 & 0,992 \\
\hline 00545004 & Barra do Corda & $-5,517$ & $-45,250$ & 838,300 & 0,171 & 10 & 0,742 & 0,993 \\
\hline 00545008 & Barra do Corda & $-5,467$ & $-45,567$ & 892,480 & 0,182 & 10 & 0,742 & 0,992 \\
\hline 00545009 & Grajaú & $-5,583$ & $-45,883$ & 863,770 & 0,166 & 10 & 0,742 & 0,993 \\
\hline 00546002 & Grajaú & $-5,817$ & $-46,467$ & 1058,770 & 0,265 & 10 & 0,742 & 0,984 \\
\hline 00546003 & Grajaú & $-5,817$ & $-46,133$ & 911,800 & 0,149 & 10 & 0,742 & 0,994 \\
\hline 00546005 & Grajaú & $-5,283$ & $-46,367$ & 890,840 & 0,145 & 10 & 0,742 & 0,994 \\
\hline 00546006 & Grajaú & $-5,604$ & $-46,239$ & 891,660 & 0,155 & 10 & 0,742 & 0,994 \\
\hline 00546007 & Sítio Novo & $-5,884$ & $-46,702$ & 934,980 & 0,139 & 10 & 0,742 & 0,995 \\
\hline
\end{tabular}




\begin{tabular}{|c|c|c|c|c|c|c|c|c|}
\hline 00547000 & Imperatriz & $-5,535$ & $-47,478$ & 905,520 & 0,156 & 10 & 0,742 & 0,994 \\
\hline 00547001 & Imperatriz & $-5,533$ & $-47,483$ & 992,660 & 0,178 & 10 & 0,742 & 0,992 \\
\hline 00547003 & Montes Altos & $-5,833$ & $-47,067$ & 797,260 & 0,170 & 10 & 0,742 & 0,993 \\
\hline 00547005 & Buritirama & $-5,594$ & $-47,019$ & 935,410 & 0,172 & 10 & 0,742 & 0,993 \\
\hline 00643011 & Barão de Grajaú & $-6,610$ & $-43,401$ & 1012,750 & 0,156 & 10 & 0,742 & 0,994 \\
\hline 00643012 & Passagem Franca & $-6,176$ & $-43,778$ & 912,010 & 0,170 & 10 & 0,742 & 0,993 \\
\hline 00643013 & Barão de Grajaú & $-6,762$ & $-43,026$ & 831,960 & 0,135 & 10 & 0,742 & 0,995 \\
\hline 00644000 & Mirador & $-6,383$ & $-44,983$ & 952,140 & 0,124 & 10 & 0,742 & 0,996 \\
\hline 00644001 & Colinas & $-6,033$ & $-44,250$ & 923,850 & 0,171 & 10 & 0,742 & 0,993 \\
\hline 00644003 & Colinas & $-6,028$ & $-44,254$ & 958,740 & 0,157 & 10 & 0,742 & 0,994 \\
\hline 00644004 & Mirador & $-6,517$ & $-44,633$ & 971,400 & 0,108 & 10 & 0,742 & 0,999 \\
\hline 00644006 & Mirador & $-6,367$ & $-44,367$ & 790,500 & 0,145 & 10 & 0,742 & 0,995 \\
\hline 00644007 & Mirador & $-6,369$ & $-44,359$ & 870,160 & 0,155 & 10 & 0,742 & 0,994 \\
\hline 00644012 & Mirador & $-6,010$ & $-44,344$ & 841,590 & 0,148 & 10 & 0,742 & 0,994 \\
\hline 00644013 & Pastos Bons & $-6,667$ & $-44,317$ & 872,970 & 0,162 & 10 & 0,742 & 0,993 \\
\hline 00644015 & Mirador & $-6,067$ & $-44,714$ & 967,390 & 0,182 & 10 & 0,742 & 0,992 \\
\hline 00644017 & Colinas & $-6,033$ & $-44,233$ & 946,460 & 0,149 & 10 & 0,742 & 0,994 \\
\hline 00645000 & Mirador & $-6,517$ & $-45,617$ & 844,110 & 0,129 & 10 & 0,742 & 0,995 \\
\hline 00645001 & Barra do Corda & $-6,283$ & $-45,217$ & 994,490 & 0,167 & 10 & 0,742 & 0,993 \\
\hline 00645002 & Barra do Corda & $-6,002$ & $-45,394$ & 1012,050 & 0,172 & 10 & 0,742 & 0,993 \\
\hline 00645003 & Loreto & $-6,844$ & $-45,109$ & 1564,590 & 0,323 & 10 & 0,742 & 0,978 \\
\hline 00646000 & Sítio Novo & $-6,233$ & $-46,467$ & 972,300 & 0,143 & 10 & 0,742 & 0,995 \\
\hline 00646001 & Grajaú & $-6,333$ & $-46,050$ & 879,020 & 0,142 & 10 & 0,742 & 0,995 \\
\hline 00646002 & Fortaleza dos Nogueiras & $-6,900$ & $-46,167$ & 880,640 & 0,192 & 10 & 0,742 & 0,991 \\
\hline 00646003 & Porto Franco & $-6,633$ & $-46,883$ & 721,440 & 0,215 & 10 & 0,742 & 0,989 \\
\hline 00646005 & Grajaú & $-6,818$ & $-46,334$ & 956,310 & 0,170 & 10 & 0,742 & 0,993 \\
\hline 00646006 & Grajaú & $-6,193$ & $-46,269$ & 1023,760 & 0,172 & 10 & 0,742 & 0,993 \\
\hline 00744000 & São Félix de Balsas & $-7,081$ & $-44,813$ & 793,600 & 0,130 & 10 & 0,742 & 0,995 \\
\hline 00745000 & Balsas & $-7,483$ & $-45,133$ & 858,030 & 0,136 & 10 & 0,742 & 0,995 \\
\hline 00745001 & Loreto & $-7,083$ & $-45,133$ & 756,480 & 0,143 & 10 & 0,742 & 0,995 \\
\hline 00745004 & Sambaíba & $-7,139$ & $-45,348$ & 886,750 & 0,153 & 10 & 0,742 & 0,994 \\
\hline 00745005 & São Raimundo das Mangabeiras & $-7,375$ & $-45,608$ & 1028,730 & 0,156 & 10 & 0,742 & 0,994 \\
\hline 00746001 & Balsas & $-7,533$ & $-46,033$ & 931,110 & 0,231 & 10 & 0,742 & 0,988 \\
\hline 00746002 & Riachão & $-7,667$ & $-46,467$ & 898,880 & 0,120 & 10 & 0,742 & 0,996 \\
\hline 00746003 & Carolina & $-7,750$ & $-46,883$ & 746,790 & 0,148 & 10 & 0,742 & 0,994 \\
\hline 00746005 & Balsas & $-7,533$ & $-46,033$ & 860,800 & 0,183 & 10 & 0,742 & 0,992 \\
\hline 00746006 & Balsas & $-7,523$ & $-46,034$ & 928,110 & 0,155 & 10 & 0,742 & 0,994 \\
\hline 00746007 & Balsas & $-7,929$ & $-45,989$ & 971,180 & 0,156 & 10 & 0,742 & 0,994 \\
\hline 00746008 & Riachão & $-7,158$ & $-46,554$ & 810,960 & 0,154 & 10 & 0,742 & 0,994 \\
\hline
\end{tabular}




\begin{tabular}{llccccccc}
00746009 & Balsas & $-7,331$ & $-46,308$ & 821,490 & 0,145 & 10 & 0,742 & 0,994 \\
00747000 & Carolina & $-7,323$ & $-47,464$ & 1002,080 & 0,158 & 10 & 0,742 & 0,994 \\
00747003 & Carolina & $-7,333$ & $-47,467$ & 976,790 & 0,133 & 10 & 0,742 & 0,995 \\
00747004 & Carolina & $-7,717$ & $-47,217$ & 892,070 & 0,146 & 10 & 0,742 & 0,994 \\
00845005 & Alto Parnaíba & $-8,821$ & $-45,968$ & 907,820 & 0,153 & 10 & 0,742 & 0,994 \\
00846000 & Balsas & $-8,967$ & $-46,867$ & 824,900 & 0,144 & 10 & 0,742 & 0,995 \\
00846001 & Balsas & $-8,500$ & $-46,467$ & 848,400 & 0,148 & 10 & 0,742 & 0,994 \\
00846002 & Balsas & $-8,167$ & $-46,233$ & 814,520 & 0,127 & 10 & 0,742 & 0,996 \\
00846003 & Alto Parnaíba & $-8,917$ & $-46,150$ & 882,880 & 0,157 & 10 & 0,742 & 0,994 \\
00846004 & Balsas & $-8,717$ & $-46,717$ & 856,840 & 0,170 & 10 & 0,742 & 0,993 \\
00846005 & Alto Parnaíba & $-8,813$ & $-46,078$ & 847,810 & 0,150 & 10 & 0,742 & 0,994 \\
00945010 & Alto Parnaíba & $-9,100$ & $-45,933$ & 977,460 & 0,163 & 10 & 0,742 & 0,993 \\
00946000 & Alto Parnaíba & $-9,300$ & $-46,700$ & 845,470 & 0,204 & 10 & 0,742 & 0,990 \\
00946001 & Alto Parnaíba & $-9,167$ & $-46,200$ & 776,960 & 0,163 & 10 & 0,742 & 0,993 \\
00946002 & Alto Parnaíba & $-9,450$ & $-46,300$ & 711,050 & 0,173 & 10 & 0,742 & 0,993 \\
00144002 & Cururupu & $-1,826$ & $-44,868$ & 1150,270 & 0,147 & 10 & 0,742 & 0,994 \\
00644014 & Benedito Leite & $-6,817$ & $-44,650$ & 682,810 & 0,155 & 10 & 0,742 & 0,994 \\
00443011 & Codó & $-4,421$ & $-43,649$ & 1073,250 & 0,148 & 10 & 0,742 & 0,994 \\
00544002 & Presidente Dutra & $-5,250$ & $-44,517$ & 982,880 & 0,120 & 10 & 0,742 & 0,996 \\
00546000 & Amarante do Maranhão & $-5,567$ & $-46,767$ & 1119,700 & 0,134 & 10 & 0,742 & 0,995 \\
00245005 & Pinheiro & $-2,517$ & $-45,083$ & 862,180 & 0,151 & 10 & 0,742 & 0,994 \\
00344004 & Cantanhede & $-3,633$ & $-44,376$ & 1080,190 & 0,155 & 10 & 0,742 & 0,994 \\
00245011 & Santa Helena & $-2,274$ & $-45,271$ & 1016,480 & 0,229 & 10 & 0,742 & 0,988 \\
00244002 & São José do Ribamar & $-2,550$ & $-44,067$ & 976,340 & 0,169 & 10 & 0,742 & 0,993 \\
00244006 & São Luís & $-2,883$ & $-44,350$ & 1205,310 & 0,163 & 10 & 0,742 & 0,993 \\
00244007 & São Luís & $-2,533$ & $-44,300$ & 1089,680 & 0,138 & 10 & 0,742 & 0,995 \\
00245006 & Santa Helena & $-2,233$ & $-45,300$ & 1579,790 & 0,207 & 10 & 0,742 & 0,990 \\
\hline & & & & & & & &
\end{tabular}

Os valores dos parâmetros $\boldsymbol{K}$ e $\boldsymbol{a}$ apresentam grande variação dentre as estações estudadas. O parâmetro $\boldsymbol{K}$ apresenta maior amplitude de variação, proporcionando o menor valor $(642,54)$ para a estação 0034201 localizada no município de Santa Quitéria do Maranhão, a Leste do Estado. Já, o maior valor calculado para esse parâmetro foi de 1749,44 para a estação 00344012 (Itapecuru Mirim) localizada a Oeste do Maranhão. Nesse mesmo sentido, o parâmetro $\boldsymbol{a}$ apresentou amplitude de 0,215 , com menor valor $(0,108)$ na estação 00644004, município de Mirador e o maior valor observado foi de 0,323 , na estação 00645003 , localizada no município de Loreto (Quadro 2).

Essa variação é justificada, pela distribuição espacial nos valores de precipitação das chuvas nas distintas localidades do estado do Maranhão, tendo em vista sua dimensão e marcada influencia climática. Por sua vez, essa variabilidade reforça a grande importância na obtenção das relações intensidade-duração-frequência para cada local específico, revelando que seus valores estão diretamente ligados à distribuição das chuvas. Em estudos realizados em outras regiões do País 
(OLIVEIRA et al., 2005; SANTOS et al., 2009; ARAGÃO et al., 2013; CAMPOS et al., 2014) também foi verificado uma grande variação nos valores dos parâmetros $\boldsymbol{K}$ e $\boldsymbol{a}$. Campos et al. (2014) deduziram que existe uma relação de interação entre os parâmetros $\boldsymbol{K}$ e $\boldsymbol{a}$, que influencia diretamente em suas estimativas. No entanto, a combinação desses parâmetros geralmente resulta em bons modelos de IDF. Quando se utiliza o modelo de regressão linear os parâmetros de ajuste $\boldsymbol{b}$ e $\boldsymbol{c}$ da IDF tendem a se manter constantes, esse fato é justificado devido aos modelos de calculo usados nas planilhas após o ajuste para primeira ou equação realizarem o ajuste apenas nos parâmetros $\boldsymbol{K}$ e $\boldsymbol{a}$.

Ao se utilizar os parâmetros ajustados para estimar a intensidade máxima pluviométrica, com duração de 10 min. e período de retorno de 10 anos, pode-se observar o comportamento das precipitações máximas estimadas em todo o estado do Maranhão (Figura 2). Nestas condições, as maiores intensidades máximas se concentraram a Norte, Noroeste e Centro-Norte do Estado. Campos et al. (2014), estudando chuvas intensas no Piauí, observaram que as chuvas máximas tenderam a aumentar das regiões Sul e Leste piauiense, para a região Norte, em direção à Amazônia, região brasileira essa que apresenta clima quente e úmido. Essa distribuição das intensidades máximas estimadas corrobora com a distribuição espacial das precipitações no estado do Maranhão (PINTO et al., 2011).

$\mathrm{Na}$ espacialização do parâmetro $\boldsymbol{K}$ (Figura 3A), observa-se que os maiores valores estão distribuídos de forma mais concentrados na região Norte e Noroeste maranhense. Isso ocorre em função dos municípios apresentarem as maiores intensidades de precipitações pluviométricas. Comportamento semelhante foi estudado por Guedeset al. (2010)

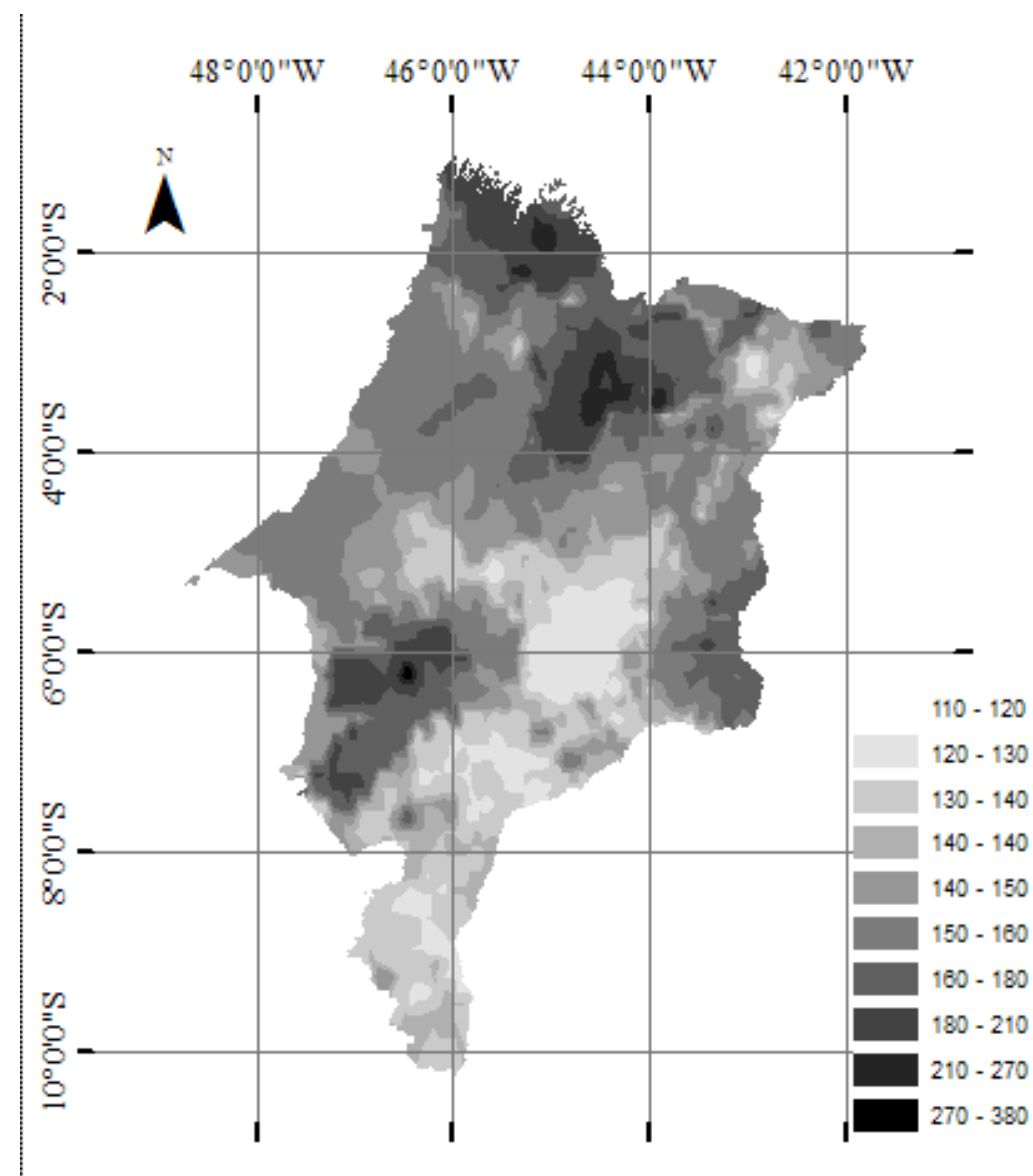

Figura 2. Espacialização das intensidades máximas estimadas para período de retorno de 10 anos com os parâmetros da IDF ajustados por regressão linear e duração de 10 min.

\section{REVENG}




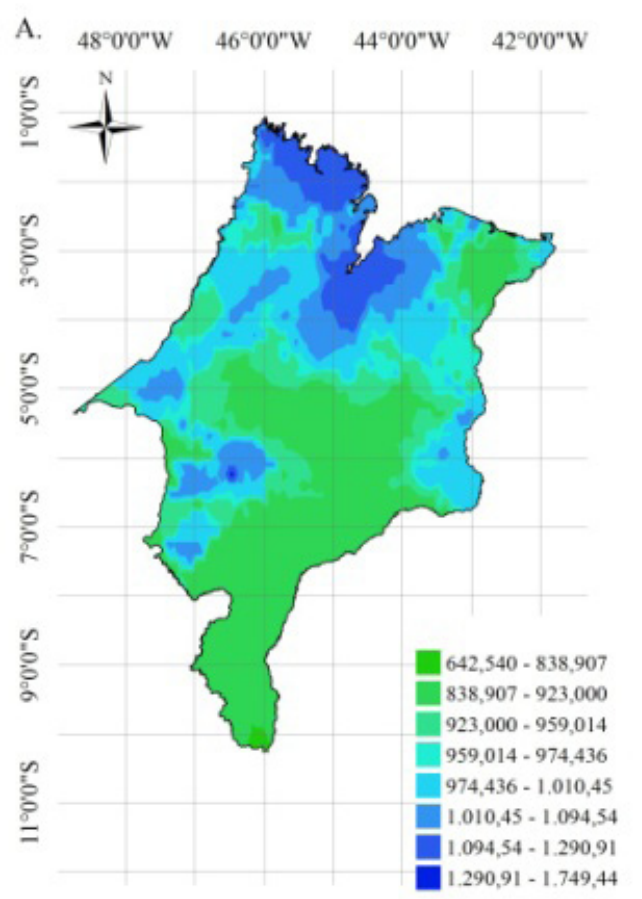

B.

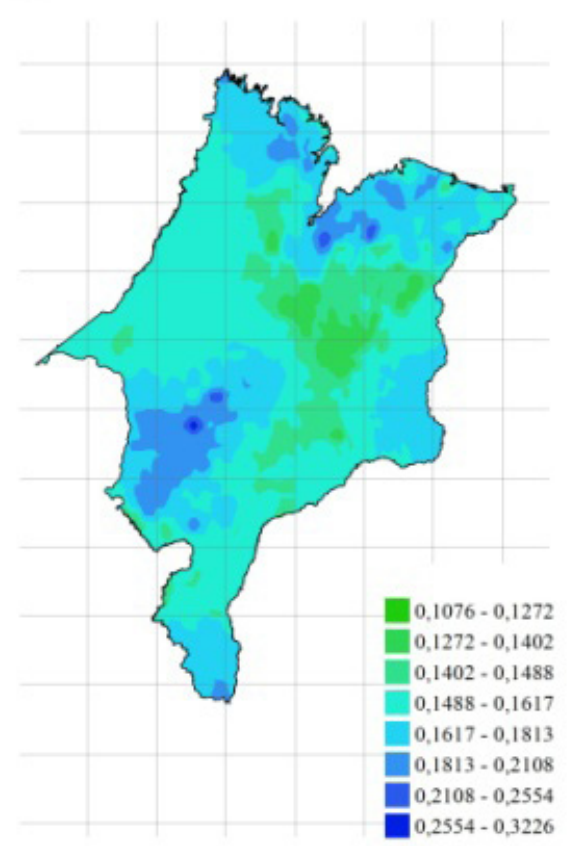

Figura 3. Espacialização do parâmetro $\boldsymbol{K}(\mathrm{A})$ e $\boldsymbol{a}(\mathrm{B})$.

e Campos et al. (2014), no Piauí, indicando que esse fenômeno está relacionado à distribuição espacial das chuvas, onde os maiores valores de pluviosidade ocorrem na região Norte e os menores na região Central, região Semiárida e no Sul do Estado.

Da mesma forma, o parâmetro $\boldsymbol{a}$ (Figura 3B) tem seus maiores valores observados nas regiões Norte e Noroeste, semelhante ao $\boldsymbol{K}$. Entretanto, nos municípios de Alto Parnaíba, Balsa e Tasso Fragoso, mais a Sudoeste do Maranhão, o parâmetro $\boldsymbol{a}$ apresentou comportamento distinto do parâmetro $\boldsymbol{K}$, que aumentou da região Leste em sentido à Amazônia. Os valores do parâmetro $\boldsymbol{b}$ e $c$ não apresentaram variação. Esse fato ocorreu em função da metodologia utilizada, pois a linearização fixa os valores desses dois parâmetros, o que também foi observado por meio dos resultados encontrados por Mello e Silva (2005).

Nesse mesmo sentido, a distribuição dos valores do parâmetro $\boldsymbol{K}$ apresenta correlação positiva com os valores de intensidade máximas estimadas (Figura 4), corroborando a distribuição espacial deste parâmetro em todo o estado do Maranhão.

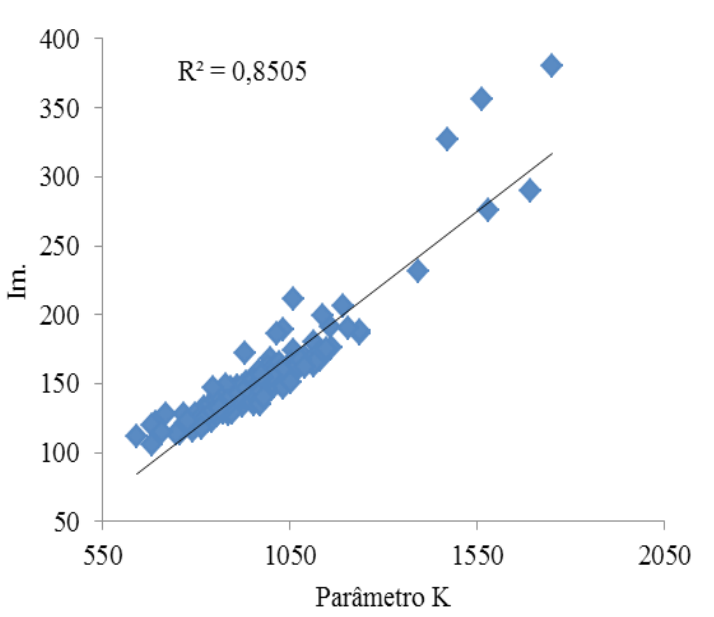

Figura 4. Correlação entre os valores de intensidades máximas estimadas (Im) e os valores do parâmetro $\boldsymbol{K}$.

\section{CONCLUSÕES}

- As equações de chuvas intensas apresentam bom ajuste, com $99,7 \%$ das equações proporcionando valores de $\mathrm{r}^{2}$ superior a 0,98 ;

- O parâmetro $\boldsymbol{K}$ apresentouos maiores valores, distribuídos de forma mais concentrados nas 
regiões Norte e Centro-Norte maranhense, em municípios próximos ao bioma Amazônia;

- Os maiores valores de intensidade máxima estimada foram observados nas regiões Norte, Noroeste e Centro-Norte do estado do Maranhão.

\section{REFERÊNCIAS BIBLIOGRÁFICAS}

ANA - Agência Nacional de Águas. Hidroweb Sistemas de Informações Hidrológicas. 2012. Disponível em: < $\quad$ http://hidroweb.ana.gov.br $>$. Acesso em: 02 maio 2014.

ARAGÃO, R.; SANTANA, G.R.; COSTA, C.E.F.F.; CRUZ, M.A.S.; FIGUEIREDO, E.E.; SRINIVASAN, V.S. Chuvas intensas para o estado de Sergipe com base em dados desagregados de chuva diária. Revista Brasileira de Engenharia Agrícola e Ambiental, v.17, n.3, p.243-252, 2013.

CAMPOS, A.R.; SANTOS, G.G.; SILVA, , J.B.L.; IRENE FILHO, J.; LOURA, D.S. Equações de intensidade-duração-frequência de chuvas para o estado do Piauí. Revista Ciência Agronômica, v.45, n.3, p.488-498, 2014.

CECÍlIO, R.A.; XAVIER, A.C.; PRUSKI, F.F.; HOLLANDA, M.P.; PEZZOPANE, J.E.M. Avaliação de interpoladores para os parâmetros das equações de chuvas intensa no Espirito Santo. Ambi-Agua, v.4, n.3 p.82-92, 2009.

CETESB - Companhia de tecnologia de saneamento ambiental. Drenagem urbana:manual de projeto. São Paulo: DAEE/CETESB, 1986. $466 \mathrm{p}$.

DAMÉ, R.C.F.; TEIXEIRA, C.F.A; TERRA, V.S.S. Comparação de diferentes metodologias para estimativa de curvas intensidade-duraçãofrequência para pelotas - RS. Revista Engenharia Agrícola, v.28, n.2, p.245-255, 2008.

DENARDIN, J.; FREITAS, P.L. Características fundamentais da chuva no Brasil. Pesquisa Agropecuária Brasileira, v.17, n.1, p.1409-1416, 1982.
GUEDES, R.V.S.; LIMA, F.J.L.; AMANAJÁS, J.C.; BRAGA, C.C. Análise em componentes principais da precipitação pluvial no estado do Piauí e agrupamento pelo método de ward. Revista de Geografia, v.27, n.1, p.208-233, 2010.

MELLO, C.R.; LIMA L.M.; SILVA, A.M.; MELLO, J.M.; OLIVEIRA, M.S. Kriging and inverse-squaredistance for the interpolation of rainfall equation parameters. Revista Brasileira de Ciência do Solo,v.27,p.925-933, 2003.

MELLO, C.R.; SILVA, A.M. Métodos estimadores dos parâmetros da distribuição de Gumbel e sua influencia em estudos hidrológicos de projeto. Irriga, v.10, n.4, p.318-334, 2005.

MORUZZI, R.B.; OLIVEIRA, S.C. Relação entre intensidade, duração e frequência de chuvas em Rio Claro, SP: métodos e aplicação. Teoria e Prática da Engenharia Civil, v.9, n.13, p.59-68, 2009.

OLIVEIRA, L.F.C.; CORTÊS, F.C.; WEHR, T.R.; BORGES, L.B.; SARMENTO, P.H.P.; GRIEBELER, N.P. Intensidade-duraçãofrequência de chuvas intensas para algumas localidades no estado de Goiás e Distrito Federal. Pesquisa Agropecuária Tropical, v.35, n.1, p.13$18,2005$.

PFAFSTETTER, O. Chuvas intensas no Brasil. Brasília: Departamento Nacional de Obras e Saneamento, 1957. 246p.

PINTO, E.J.A.; AZAMBUJA, A.M.S. de; FARIAS, J.A.M.; SALGUEIRO, J.P.B.; PICKBRENNER, K. (Coords.). (2011). Atlas pluviométrico do Brasil: isoietas mensais, isoietas trimestrais, isoietas anuais, meses mais secos, meses mais chuvosos, trimestres mais secos, trimestres mais chuvosos. Brasília: CPRM, 1 DVD. Disponível em: <http://www.cprm.gov.br/publique/media/ Isoietas_Totais_Anuais_1977_2006.pdf $>$. Acesso em: 01 agosto. 2015

RODRIGUES, J.O; ANDRADE, E.M.; OLIVEIRA, T.S.; LOBATO, F.A. Equações de 
intensidade-duração-frequência de chuvas para as localidades de Fortaleza e Pentecoste, Ceará. Scientia Agraria, v.9, p.511-519, 2008.

SANTOS, G.G.; FIGUEIREDO, C.C.; OLIVEIRA, L.F.C.; GRIEBELER, N.P. Intensidade-duraçãofrequência de chuvas para o Estado de Mato Grosso do Sul. Revista Brasileira de Engenharia Agrícola e Ambiental, v. 13, p.899-905, 2009.
SANTOS, G.G.; NORI, P.G.; OLIVEIRA, L.F.C. Chuvas intensas relacionadas à erosão hídrica. Revista Brasileira de Engenharia Agrícola e Ambiental, v.14, n.2, p.115-123, 2010.

VILlELA, S.M.; MATTOS, A. Hidrologia aplicada. São Paulo: McGraw Hill do Brasil, 1975. 245p. 